Rev. Elev. Méd. vét. Pays trop., 1978, 31 (3) : 293-313.

\title{
Expérience de pathologie comparée entre bovins zébus et Ndama soumis à l'infection naturelle par des trypanosomes pathogènes
}

\author{
par Saydil M. TOURE $\left({ }^{* *}\right)$, Arona GUEYE $\left({ }^{* *}\right)$, Mamadou SEYE $\left({ }^{* *}\right)$, \\ Mohamed A. BA $\left({ }^{* *}\right)$, A. MANE $(* *)$ et al.
}

RÉSUMÉ

\begin{abstract}
Les auteurs ont étudié l'évolution des trypanosomiases à $T$. vivax et $T$. congolense sur des zébus et Ndama n'ayant jamais hébergé ces parasites, après les avoir exposés à l'infestation naturelle dans une région riche en Glossina morsitans submorsitans et $G$. palpalis gambiensis.

Ils décrivent, de façon comparative, l'évolution des affections ainsi provoquées et les lésions observées, avec comme conclusion essentielle que si les zébus sont très sensibles à l'infection trypanosomienne, les Ndama qui n'ont jamais été, au préalable, en contact avec des glossines infestées le sont aussi, à un certain degré. Certains Ndama présentent une résistance remarquable et ces animaux méritent une étude immunologique très poussée ainsi que des études génétiques car de tels sujets peuvent être retenus dans la sélection ou le croisement pour créer des souches naturellement et totalement trypano-résistantes.
\end{abstract}

Dans la pathologie des trypanosomiases animales, les données sont très nombreuses mais il arrive souvent que des questions ne trouvent pas de réponses satisfaisantes. Il en est ainsi en matière de pathologie comparée pour connaître des différences entre le rôle pathogène des diverses espèces de trypanosomes et pour expliquer la sensibilité respective de différentes races animales. En ce qui concerne plus particulièrement les bovins, les zébus et les taurins Ndama doivent faire l'objet de comparaisons plus précises pour déterminer les causes de leur différence de comportement, par tous reconnue, à l'égard des trypanosomiases. C'est à cette fin

(*) Etude financée par le Laboratoire international de Recherches sur les Maladies animales (International Laboratory for Research on Animal Diseases. ILRAD. Nairobi).

(**) Institut sénégalais de Recherches agricoles (I. S. R. A.). Laboratoire national de l'Elevage et de Recherches vétérinaires. Dakar.

(***) Direction de la Santé et des Productions animales, Sénégal. qu'une convention de recherche a été passée entre le Laboratoire international de Recherches sur les Maladies animales (International Laboratory for Research on Animal Diseases, I. L. R. A. D.) de Nairobi et le Laboratoire national de l'Elevage et de Recherches vétérinaires de Dakar, Sénégal.

Les travaux ont duré 8 mois, de mai à décembre 1977. Les principales étapes et les résultats obtenus sont analysés ci-après.

\section{MATÉRIEL ET MÉTHODES}

Des animaux achetés en région indemne de trypanosomiase ( 20 zébus et $29 \mathrm{Ndama}$ ) ont été placés dans une région infestée de glossines. Avant expérimentation, ils ont fait l'objet d'analyses de routine pour évaluer leur état de santé. Une fois sur le site expérimental, ces bovins sont suivis au jour le jour et l'étude de 
divers paramètres biologiques est faite périodiquement. Les animaux éventuellement morts ou agonisants sont autopsiés et les lésions précisées. Voici les détails du protocole.

\section{Animaux d'expérience}

Ils sont au nombre de 49 , soit :

- 18 Ndama de la région de Diourbel (lot N1) ;

- 7 Ndama de Bargny et de Wayembam, région du Cap-Vert (lot N2);

- 4 métis Ndama-Zébu, du Centre national de Recherches agronomiques de Bambey (C. N. R. A.) : lot ZN ;

- 10 zébus de Diourbel (lot $\mathrm{Z} 1$ ) ;

- 10 zébus du Centre de Recherches zootechniques de Dahra (C. R. Z.) : lot Z2.

Les sept Ndama achetés auprès des éleveurs de Bargny et de Wayembam (région du CapVert) ainsi que les 4 métis du Centre national de Recherches agronomiques de Bambey (C. N. R. A.) n'ont très certainement jamais été parasités par des trypanosomes pathogènes. Les métis en question sont très voisins des Ndama (13/16 de Ndama). L'ensemble des zébus est aussi considéré comme n'ayant jamais été trypanosomé. Par contre, les autres Ndama (N1) ont un passé mal défini et les analyses faites postérieurement à l'achat montrent avec évidence que quelques-uns d'entre eux ne sont pas indemnes. Toutefois cette constatation ne compromet pas la valeur des résultats obtenus et elle est même de quelque intérêt dans nos comparaisons.

L'âge et le sexe des animaux sont indiqués ci-dessous :

N1 : mâles castrés de 5 à 8 ans

N2 : mâles castrés de 3 à 5 ans

ZN : femelles : génisses de 8 à 10 ans

Z1 : mâles castrés (sauf 1) de 4 à 6 ans

Z2 : génisses gestantes de 5 ans.

Avant expérimentation, l'ensemble des lots a subi plusieurs analyses lors de la quarantaine au laboratoire : coprologie parasitaire, recherche d'hémoparasites, recherche spécifique de trypanosomes, hématocrite, numération globulaire, formule leucocytaire, évaluation des globulines totales, évaluation des IgM et des IgG.

\section{Site expérimental}

Le site expérimental est localisé au village de Missira $\left(13^{\circ} 40^{\prime}\right.$ nord $-16^{\circ} 30^{\prime}$ ouest). Il a été choisi en fonction de la présence de glossines (Glossina morsitans submorsitans dans la forêt de Fathala et G. palpalis gambiensis au marigot de Néma). Ces glossines transmettent localement Trypanosoma vivax vivax, $T$. congolense congolense et $T$. brucei brucei, comme l'ont montré des enquêtes antérieures.

\section{Personnel}

Sur le site expérimental ont séjourné en permanence un ingénieur des travaux d'élevage, un aide de laboratoire et 5 manouvres et bergers. Deux docteurs vétérinaires ont assuré les liaisons hebdomadaires entre le laboratoire et le site. $\mathrm{Au}$ laboratoire même, la plupart des analyses sont réalisées par un autre ingénieur des travaux d'élevage et 2 aides.

\section{Facilités}

Pendant toute la durée d'expérimentation, un camion-laboratoire stationné sur place a permis, grâce à un générateur de courant, de procéder à la centrifugation microhématocrite et d'observer au microscope le sang des animaux. De plus, le sang récolté peut être gardé à $+4^{\circ} \mathrm{C}$ dans un réfrigérateur.

Un autre véhicule aménagé en bétaillère a assuré le transport des bovins sur le site et, en cas de nécessité, du site au Laboratoire, pour des nécropsies plus commodes.

Les liaisons hebdomadaires utilisent une voiture légère.

\section{Méthodes}

1) Examen clinique : il a lieu tous les jours ; les agents sur place notent au jour le jour les signes observés sur les animaux.

2) La température corporelle est relevée tous les 2 jours, mais plus souvent pour les animaux prostrés et parasitémiques.

3) Les ganglions préscapulaires et précruraux sont mesurés avant l'infection et en cours de maladie. 


\section{LOCALISATION DU SITE EXPERIMENTAL}

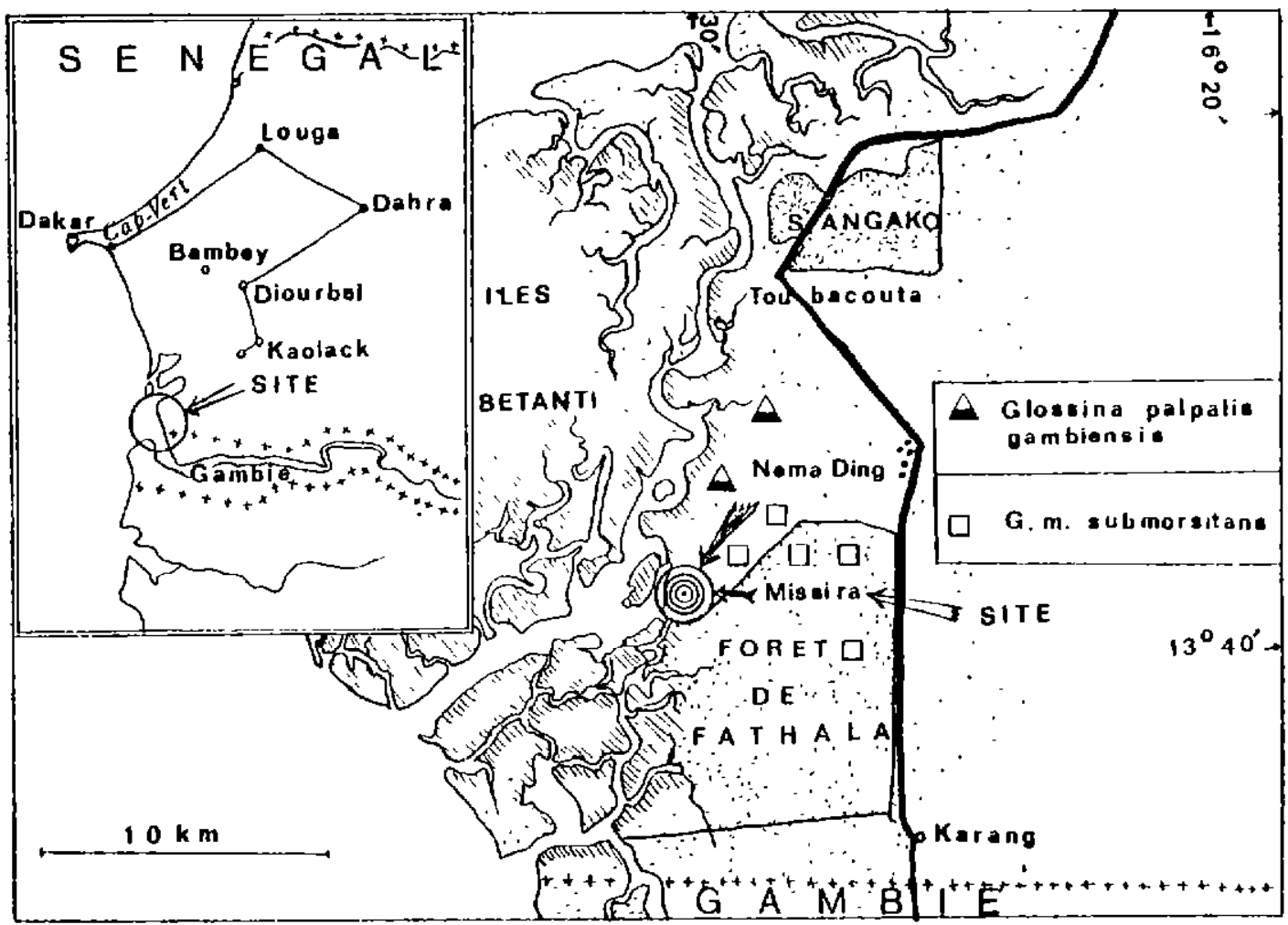

4) Parasitémie : elle est évaluée sur l'ensemble du troupeau au moins une fois par semaine: interphase de tube microhématocrite examiné sur fond noir (méthode de WOO) $(16,17)$ : en même temps sont réalisés des frottis et gouttes épaisses à colorer. En quelques occasions d'autres techniques ont été pratiquées : hémoculture en bouillon nutritif pour la mise en évidence de $T$. theileri et inoculation de sang à des souris pour la détection de $T$. brucei et $T$. congolense.

5) Hématologie: cytohématologie par évaluation du volume des cellules après centrifugation microhématocrite (PCV), au moins une fois par semaine, numération globulaire et formule leucocytaire avant et en cours de maladie ainsi qu'à la mort ; image des cellules sanguines dans le système réticulohistiocytaire. L'hémoglobine a aussi fait l'objet de mesures par la technique utilisant le cyanure de potassium.

6) Sérologie : évaluation des globulines totales et des fractions IgM et IgG. Dans le premier cas, les globulines sont précipitées par du sulfate d'ammonium, recueillies dans un réactif cuprotartrique (méthode du biuret) et dosées par colorimétrie. Dans l'évaluation des IgM et des IgG, il a été procédé à des épreuves d'immuno- diffusion sur gélose en utilisant des anti-IgM, $\mathrm{IgG}_{1}$ et $\mathrm{IgG}_{2}$ de bovins, réactifs achetés dans le commerce $\left(^{*}\right)$. Les mesures portent non sur les quantités respectives des classes d'anticorps mais sur le degré d'augmentation au cours de la maladie. Pour ce faire, nous prenons pour base une réaction correspondant à un anneau large de $1 \mathrm{~mm}$, comme étant la valeur minimale positive lisible. Un lot de sérums à une date donnée sera caractérisé par la moyenne géométrique des titres réciproques d'anticorps (Geometrical Mean of Reciprocal Titer, G. M. R. T. selon WAUGH, in : AMBROISE-THOMAS) (1) :

$$
\text { GMRT }=\operatorname{antilog} \Sigma f(\log x) / N
$$

où $\mathrm{x}$ représente les titres réciproques d'anticorps

f le nombre de sérums qui ont respectivement donné chacun de ces titres

$\mathrm{N}$ le nombre total de sérums.

C'est ce même procédé qui est suivi dans l'étude sérologique des sérums par immunofluorescence avant expérimentation et en cours d'expérience.

(*) Chemical Credentials, Miles Ltd. 
Dans les épreuves d'immunofluorescence on a utilisé un antigène Trypanosoma brucei brucei récolté sur rat et un sérum de lapin anti-globulines totales de Zébu et Ndama mélangées avant immunisation. Nous avons repris la même évaluation de la positivité que dans une publication antérieure (14) :

$$
\begin{aligned}
& +++ \text { trypanosomes présentant une fluores- } \\
& \text { cence brillante sur fond noir } \\
& ++++ \text { fluorescence très brillante. }
\end{aligned}
$$

8) Les animaux morts sont autopsiés, les lésions étudiées macroscopiquement et microscopiquement par les procédés classiques d'histologie. En certaines circonstances où les animaux étaient en décubitus prolongé, l'euthanasie tirait son indication d'une baisse matquée de température (hypothermie à $36^{\circ} \mathrm{C}$ ou moins).

9) Pour analyser les différences entre les groupes d'animaux, les procédés de calcul statistique suivent L. LISON (7). Les résultats sont tous exprimés avec une probabilité se rapportant à $\mathbf{t}=0,05$, avec des degrés de liberté numériquement petits.

10) L'observation au microscope, sur fond noir, après centrifugation, n'est pas accompagnée de numération du nombre de trypanosomes; nous nous sommes contentés d'une évaluation de la parasitémie ainsi qu'il suit :

+ au moins un trypanosome sur la préparation ou bien en moyenne 1 trypanosome dans 10 champs du microscope

++ une moyenne de 1 trypanosome dans 2 à 10 champs

+++ trypanosomes présents dans tous les champs à raison d'au moins 1

++++ parasites excessivement nombreux dans un champ et comptage difficile.

\section{ETUDES AVANT EXPÉRIMENTATION}

\section{Examen clinique}

Les animaux dans leur ensemble sont apparemment en très bonne santé, sans hypertrophie ganglionnaire ni signes morbides quelconques. Cette bonne santé de visu est cependant une notion relative qui ne cadre pas avec les investigations biologiques.

\section{Etude parasitologique}

En effet des parasites ont été décelés chez ces animaux, soit :

\section{- Parasites gastro-intestinaux}

Les bovins de tous les lots présentent un ou plusieurs des parasites suivants (le plus souvent même plusieurs) :

- Haemoncus

- Cooperia

- Oesophagostomum

- Trichostrongylus

- Bunostomum

- Strongyloides

- Eimeria.

\section{- Parasites du sang}

Les Ndama ont pour la plupart (19 sur 29) des hémoparasites mais ceux-ci, sauf dans 2 cas de trypanosomiase avérée, ne semblent pas liés à une baisse des valeurs de l'hématocrite.

Le lot de zébus, quant à lui, n'est que faiblement parasité : 3 sur 20 par Theileria mutans (hématocrite 32-40) et un seul par microfilaires de Setaria labiatopapillosa (hématocrite 33).

Les ectoparasites sont très rares sur les animaux : quelques tiques (Boophilus decoloratus et Amblyomma variegatum).

Le parasitisme des animaux d'expérience est regrettable mais à l'heure actuelle c'est un obstacle pratiquement insurmontable, à moins d'avoir des élevages spécialement destinés à fournir des sujets pour des études de pathologie.

Pour rendre les conditions aussi semblables que possible les lots ont été déparasités, pour ce qui est des helminthes, en utilisant le thiabendazole, 2 semaines avant l'expérimentation et au second mois sur le terrain. Les animaux sont aussi détiqués une fois tous les 15 jours par un acaricide (dicrotophos puis $\mathrm{HCH}$ ).

\section{Etude hématologique}

\section{Hématocrite}

En excluant les 2 Ndama trypanosomés, l'amplitude de l'hématocrite va de 32 à 58 . Les moyennes observées durant la quarantaine sont :

- $1^{\text {er }}$ groupe de Ndama (N1) : 45,7 $\mp 3,4$

- $2^{\mathrm{e}}$ groupe de Ndama (N2) : 38,2 $\mp 4,1$

- groupe de métis (ZN) : 39,5 73,9

- groupe de zébus (Z1 et Z2) : 45,7 7,8 . 
L'hématocrite des $\mathrm{N} 1$ est significativement plus élevée que celle des groupes $\mathrm{N} 2$ et $\mathrm{ZN}$, mais $\mathrm{N} 1$ et $\mathrm{ZN}$ ne présentent pas de différences significatives. Les moyennes observées sont supérieures à celles citées par O. W. SCHALM (12) et par D. FRIOT et H. CALVET (5) que nous retiendrons comme normes.

\section{Globules blancs et globules rouges}

Voici les moyennes observées dans les différents lots et se rapportant aux valeurs d'hématocrite ci-dessus.

TABLEAU N* I

\begin{tabular}{|c|c|c|c|c|c|c|}
\hline \multirow[b]{2}{*}{ Groupes } & \multicolumn{2}{|c|}{ Numê ration } & \multicolumn{4}{|c|}{ Eormule leucocytaire } \\
\hline & $\begin{array}{l}\text { G. rouges } \\
\left(\times 10^{6}\right)\end{array}$ & $\begin{array}{c}\text { G. blancs } \\
\left(\mathrm{x} 10^{3}\right)\end{array}$ & $\begin{array}{c}\text { Neutrophiles } \\
\text { p. } 100\end{array}$ & $\begin{array}{l}\text { Eosinophiles } \\
\text { p. } 100\end{array}$ & $\begin{array}{l}\text { Lymphocytes } \\
\text { p. } 100\end{array}$ & $\begin{array}{c}\text { Monocytes } \\
\text { P.100 }\end{array}$ \\
\hline Nl & $7,5 \pm 1,2$ & $6,05 \pm 0,5$ & $19 \pm 2$ & $9 \pm 3$ & $61 \pm 5$ & $11 \pm 3$ \\
\hline $\mathrm{N} 2$ & $6,35 \pm 1,4$ & $4,30 \pm 2,2$ & $40 \pm 10$ & $4 \pm 1$ & $49 \pm 8$ & $8 \pm 3$ \\
\hline $\mathrm{ZN}$ & $6,90 \pm 0,5$ & $4,60 \pm 1,3$ & $33 \pm 8$ & $11 \pm 10$ & $48 \pm 11$ & $8 \pm 3$ \\
\hline $\mathrm{zl}$ & $7,60 \pm 1,4$ & $6,66 \pm 1,2$ & $32 \pm 1$ & $7 \pm 5$ & $60 \pm 4$ & $4 \pm 2$ \\
\hline$Z 2$ & $7,70 \pm 0,9$ & $7,65 \pm 0,3$ & $36 \pm 5$ & $8 \pm 2$ & $51 \pm 5$ & $5 \pm 2$ \\
\hline
\end{tabular}

Ces données appellent les commentaires suivants :

- le nombre de globules rouges est voisin de la normale ; le calcul du volume globulaire moyen donne respectivement :

- pour N1, $60 \mathrm{fl}$

- pour N2, $51 \mathrm{fl}$

- pour ZN, $57 \mathrm{fl}$

- pour $\mathrm{Z1}$ et $\mathrm{Z} 2,59 \mathrm{fl}$;

- on constate, dans l'ensemble, une légère macrocytose, sauf dans le groupe $\mathrm{N} 2$;

- le nombre de globules blancs est aussi voisin de la normale dans les groupes $\mathrm{NI}, \mathrm{Zl}$ et $\mathrm{Z} 2$ mais il y a leucopénie dans les lots N2 et $\mathrm{ZN}$;

- sauf en $\mathrm{N} 1$, il y a notablement plus de granulocytes neutrophiles que normalement; les lymphocytes et les monocytes sont en plus grand nombre dans certains des lots, mais aucune anomalie n'est prouvée par ces mesures.

\section{Epreuves sérologiques}

Les globulines totales du sérum n'ont $\mathrm{pu}$ être analysées que durant la période d'épreuve sur le terrain.

Concernant l'IgM, on note avant expérience des valeurs relativement faibles de la M. G. T. R. pour des dilutions de $1 / 10$ à $1 / 160$ (n étant le nombre de sérums analysés) : tableau $n^{\circ}$ II.

\begin{tabular}{|l|c|c|c|c|c|c|c|c|}
\hline Groupes & $\mathrm{n}$ & 1 & $1 / 10$ & $1 / 20$ & $1 / 40$ & $1 / 80$ & $1 / 160$ & MGTR \\
\hline $\begin{array}{l}\text { N1 et } \\
\text { N2 }\end{array}$ & 20 & 1 & 0 & 6 & 5 & 6 & 2 & 38,22 \\
\hline 21 & 9 & 2 & 3 & 2 & 2 & 0 & 0 & 9,73 \\
\hline 22 & 9 & 1 & 3 & 2 & 3 & 0 & 0 & 14,33 \\
\hline
\end{tabular}

L'analyse de $1^{\prime} \operatorname{IgG}_{1}$ par la technique utilisée ne donne pas de résultats exploitables; il en est de même de $1^{\prime} \mathrm{IgG}_{2}$ pour laquelle on observe chez tous les animaux de larges anneaux de précipitation avec l'antisérum.

L'immunofluorescence, avec des sérums dilués de $1 / 20$ à $1 / 160$, ne dénonce que 4 animaux sérologiquement positifs, uniquement dans le groupe $\mathrm{NI}$; ce sont :

- les 2 bovins présentant $T$. congolense et dont les sérums réagissent jusqu'à 1/80 $(+++)$;

- 2 animaux non parasitémiques qui réagissent à $1 / 20$ et $1 / 160$ respectivement $(+++)$.

Dans ce même lot, il y a cependant plusieurs suspects sérologiques qui réagissent faiblement à des dilutions $1 / 20$ à $1 / 80$. Les groupes $Z 1, Z 2$, $\mathrm{ZN}$ et $\mathrm{N} 2$ sont négatifs au $1 / 20$ pour l'ensemble des animaux. Les normes de positivité adoptées conduisent à une M. G. T. R. nulle pour l'ensemble des lots en excluant les 2 animaux réellement parasitémiques et les deux suspects. C'est le point de départ des comparaisons ultérieures. 


\section{PHASE D'INFECTION NATURELLE}

Les données qui suivent portent sur les observations faites de mai à décembre 1977 . L'accent est mis, chaque fois que constatées, sur les différences entre les lots expérimentaux.

\section{Période de prépatence}

Elle est exprimée par le nombre de jours écoulés entre l'arrivée des animaux sur le site et l'apparition de trypanosomes dans le sang. Faute d'examens quotidiens du sang de tous les bovins, les durées obtenues peuvent comporter une erreur de 2 à 3 jours en plus. La durée de prépatence la plus courte observée est de 9 jours et elle se rapporte à un zébu du groupe $\mathrm{Z1}$, tandis que pour les Ndama cette durée est de 11 jours. Les données indiquent des différences entre les taurins Ndama au sens large et les zébus. On notera que, pour ceux-ci, la prépatence n'excède pas 30 jours tandis qu'avec les premiers elle peut atteindre plusieurs semaines voire plusieurs mois. Toutefois, quelque discernement s'impose : plusieurs des taurins ne révèlent pas de grande différence d'avec les zébus, notamment dans les lots $\mathrm{N} 2$ et $\mathrm{ZN}$, alors que d'autres, du groupe $\mathrm{N} 1$, sont restés pour la plupart assez longtemps avant de présenter une parasitémie, ou bien même n'en ont pas présenté du tout. Devant de tels cas il y a lieu de se demander si ces animaux n'ont pas eu, en fait, une trypanosomiase passée ayant entraîné un état immunitaire solide.

Il faut admettre que les Ndama qui n'ont jamais été infectés présentent une période de prépatence légèrement plus élevée que celle des zébus (voir courbes de prépatence).

\section{Parasitémie}

1) Des différentes méthodes d'étude de la parasitémie, il ressort une complémentarité nécessaire :

a) la technique de WOO, suivie d'observation microscopique sur fond noir, renseigne assez rapidement sur la nature et le degré de parasitémie ; cependant elle comporte des restrictions :

- si $T$. congolense est rare, on peut ne pas voir ce parasite ;

- l'observateur, en présence de nombreux trypanosomes dont certains sont très mobiles et d'autres peu actifs, en partie masqués par les globules rouges, peut conclure à une infection mixte $T$. vivax $T$. congolense alors qu'il s'agit de la première espèce seulement ;

b) les frottis et gouttes épaisses restent indispensables pour confirmer les espèces après coloration ;

c) l'inoculation de souris et l'hémoculture s'imposent pour déceler respectivement $T$. brucei et $T$. theileri chez les bovins.

2) La technique de WOO et l'observation sur fond noir ont été constamment pratiquées sur le terrain puis le diagnostic confirmé au Laboratoire sur lames colorées. Les résultats sont :

a) pour l'ensemble des animaux, les infections à $T$. vivax sont de loin les plus nombreuses. L'explication réside sans doute dans le fait que les glossines du gîte sont davantage parasitées par cette espèce, mais surtout que d'autres Diptères vulnérants doivent assurer une transmission non cyclique. Ainsi sur 35 infections, en première vague parasitémique, il y en a 26 à $T$. vivax, 7 à $T$. congolense et 2 mixtes à $T$. vivaxT. congolense ;

b) $T$. congolense est plus fréquente chez les Ndama (6 animaux sur 19 infectés) que chez les zébus ( 3 animaux dont 2 à infection mixte $T$. vivax- $T$. congolense sur 16), toujours en première vague parasitémique ;

c) l'intensité de la parasitémie initiale, à la première semaine où elle a été constatée, est plus marquée chez les zébus :

-11 animaux +++ ou ++++ (T. vivax $)$ lense)

-1 animal $+++(T$. vivax $)$ et $+(T$. congo-

-2 animaux + et $++(T$. vivax $)$

-1 animal $+(T$. congolense $)$ et $+(T$. vivax $)$

-1 animal $+(T$. congolense $)$;

par contre, elle l'est moins dans les lots de taurins :

-2 animaux +++ et $++++(T$. vivax $)$

-11 animaux + ou $++(T$. vivax $)$

-6 animaux + ou $++(T$. congolense $)$;

d) il apparaît que c'est $T$. vivax qui entraîne d'emblée une parasitémie très élevée, particulièrement chez les zébus et le groupe métis $\mathrm{ZN}$. $\mathrm{Ce}$ fait nous a dicté le traitement dans les lots $\mathrm{Z} 2$ et $\mathrm{ZN}$ pour éviter de perdre tous ces animaux en peu de temps. Ils ont reçu 3 traitements par le Bérénil et se sont régulièrement réinfectés au 


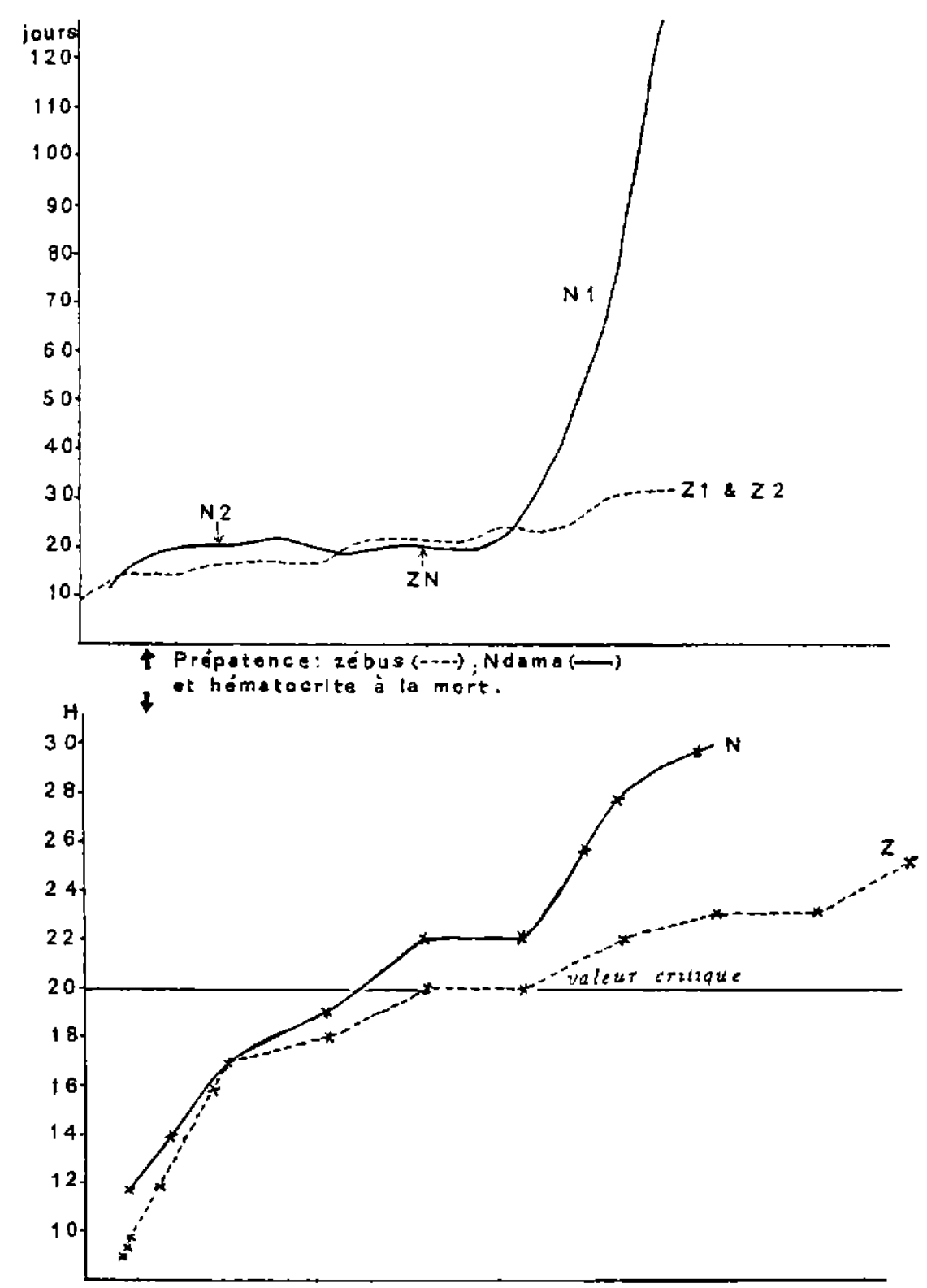

bout de 4 à 5 semaines. Ce geste est assimilable, dans une certaine mesure, à une induction de chronicité ;

e) dans 3 infections de Ndama par $T$. congolense, il y a eu, les semaines suivantes, apparition de $T$. vivax et dans une infection à $T$. vivax, apparition de $T$. congolense. Pour ces infections mixtes, l'apparition de $T$. vivax est plus constante que celle de $T$. congolense;

$f$ ) chez plusieurs Ndama, pendant les semaines et les mois qui ont suivi l'infection initiale, on constate des périodes microscopiquement apara- sitémiques, contrairement aux zébus chez qui l'infection persiste. De plus, ces derniers ont toujours beaucoup plus de trypanosomes que les Ndama.

3) On peut retenir que les trypanosomes en cause dans les types de maladie étudiés sont $T$. vivax et $T$. congolense car l'inoculation de souris au troisième mois de l'expérimentation n'a révélé qu'une seule infection due à $T$. brucei (bovin Ndama présentant à la fois $T$. vivax$T$. congolense- $T$. brucei et mort après 180 jours d'épreuve). Trois animaux seulement ont présenté $T$. theileri, non pris en considération ici. 


\section{Température}

Aux accès parasitémiques correspondent, le plus souvent, une élévation de la température rectale. Ce fait est classiquement admis. Il est intéressant de noter toutefois que l'hyperthermie est souvent plus marquée dans les lots de zébus (une observation à $41^{\circ} 3$ ) que dans ceux de Ndama.

La moyenne des températures se rapportant aux accès est de $39^{\circ} 4 \mp 0,2$ chez les zébus et $38^{\circ} 8 \mp 0,2$ chez les Ndama (moyenne assez voisine de la normale $38^{\circ} 6$ ) ; la différence de moyenne dans les deux races est faible mais toutefois significative et, sans doute, est en corrélation avec les différences constatées dans l'intensité de la parasitémie.

Chez les animaux qui succombent à la maladie, la mort est précédée par une hypothermie $\left(36^{\circ}\right.$ ou moins).

\section{Hématocrite}

Il y a une corrélation absolue entre la baisse de l'hématocrite et l'infection trypanosomienne, évidente ou cryptique.

1) Quel que soit le lot pris cn considération, une baisse de la moyenne globale de l'hématocrite survient dès les deux premières semaines, même si les trypanosomes n'apparaissent que sur quelques animaux.

En considérant la première vague parasitémique, se rapportant à l'ensemble des animaux trypanosomés au cours de l'expérimentation, l'hématocrite est de $27,8 \mp 3,2$ soit donc une baisse accusée par rapport aux valeurs initiales.

2) Au début de la parasitémie, il n'y a pas de différences significatives entre les groupes $(27,9 \mp 3,4$ pour les Ndama et $28,8 \mp 3,1$ pour les zébus).

3) On note dans ces valeurs une légère supériorité des zébus mais elle est factice car ce sont les trypanosomes en cause qui entraînent les différences :

- aux infections dues à $T$. vivax correspond une hématocrite moyenne de $29,9 \mp 3$ tandis qu'avec $T$. congolense, celle-ci est de 24,3 $\mp 8,2$;

- en conséquence, apparemment, l'anémie due à $T$. congolense est plus sévère que celle due à $T$, vivax;

- et, précisément, les Ndama hébergent ici davantage ce parasite.
4) Passée la première vague parasitémique, la baisse de l'hématocrite s'accentue chez les animaux voués à la mort mais se rétablit périodiquement autour de valeurs normales chez certains Ndama. En séparant dans chaque groupe, au cours d'une observation donnée, les animaux parasitémiques de ceux qui ne le sont pas, on note le plus souvent une différence significative. A titre d'exemple :

\section{- chez les Ndama}

- à la $3^{e}$ semaine d'expérience :

- ensemble des animaux : $30,2 \mp 7,8$

- non trypanosomés : $\quad 32,2 \mp 3,5$

- trypanosomés : $\quad 24,8 \mp 7,8$

- à la $4^{e}$ semaine d'expérience

— ensemble des animaux : 25,8 $\mp 2$

一 non trypanosomés : $\quad 27,3 \mp 3,3$

- trypanosomés : $\quad 22,8 \mp 4,5$

- chez les zébus à la $5^{\mathrm{e}}$ semaine d'expérience :

- ensemble des animaux : 28,8 $\mp 4,7$

- non trypanosomés : $\quad 32,1 \mp 7,9$

- trypanosomés : $\quad 24,8 \mp 11,8$

(les valeurs des semaines suivantes ne sont pas prises en considération, certains des zébus ayant reçu un traitement).

5) Le groupe de Ndama N1, après 4 semaines sur le terrain, se comporte de façon admirable en ne présentant que 2 ou 3 sujets parasitémiques de temps en temps; les moyennes de l'hématocrite, au long des semaines, sont toujours élevées : $44 \mp 4$ avant expérimentation puis sur le terrain successivement $31,1 \mp 3,8$; $26,7 \mp 3,1 ; 33,4 \mp 3 ; 31,7 \mp 3,4 ; 30,5 \mp 5,5$; $34,3 \mp 2,9 ; 36,8 \mp 2,8 ; 33,7 \mp 3,1 ; 37,3 \mp 2,6$; $37,7 \mp 3,4 ; 37,4 \mp 3,4 ; 39,4 \mp 2,8$ etc. 11 est remarquable que les zébus, même traités, ne donnent pas de valeurs qui atteignent celles de ces Ndama.

6) Indépendamment du parasite en cause, l'hématocrite au moment de la mort est significativement un peu plus élevée chez les Ndama $(m=22,75 \mp 6,14)$ que chez les zébus $(\mathrm{m}=19,77 \mp 3,7)$. Ce point sera discuté plus loin.

\section{Numération globulaire, hémoglobine et formule leucocytaire}

Les données obtenues sont si fragmentaires qu'elles ne permettent pas une comparaison entre 
zébus et Ndama. Cela tient essentiellement à l'impossibilité d'analyser valablement des échantillons de sang dont beaucoup sont hémolysés en cours de transport. Cependant, les valeurs de l'hématocrite mentionnées ci-dessus sont suffisamment éloquentes. On peut même dire que, pour les deux races, la maladie est accompagnée, dans les cas aigus, d'une baisse notable des globules rouges et assez similaire $\left(2,55 \times 10^{6}\right.$ observé chez un zébu et $2,80 \times 10^{6}$ chez un Ndama comme valeurs minimales). Les dernières analyses, faites sur les Ndama seulement, montrent une diminution de l'hémoglobine chez les trypanosomés $(7,65 \mathrm{~g} / 100 \mathrm{ml} \mp 1,78$ contre $10,6 \mathrm{~g} / 100 \mathrm{ml} \mp 2 \mathrm{chez}$ les animaux non parasitémiques; la différence est significatıve). Pour la même série d'analyse, la concentration corpusculaire moyenne en hémoglobine est respectivement égale à 0,26 et 0,28 . 11 y a hypochromie aussi bien chez les parasités que chez les autres, qui ne révèlent rien.

Les formules leucocytaires traduisent, dans les premières semaines d'infection, une baisse des granulocytes polynucléaires neutrophiles et une augmentation des éosinophiles et des lymphocytes chez les Ndama; les zébus, par contre, voient leurs neutrophiles augmenter et les lymphocytes diminuer.

La baisse des éosinophiles est remarquable chez les Ndama morts trypanosomés. Les autres comparaisons sur ces animaux morts ne révèlent de différences significatives que pour les neutrophiles qui sont en proportion plus élevée chez les zébus.

\section{Globulines}

Durant les premières semaines et les premiers mois de séjour sur le terrain, il est constaté une augmentation progressive des quantités de globulines totales du sérum.

On notera pour mémoire que les dernières valeurs obtenues chez les zébus sont consécutives au premier traitement (augmentation notable). Les semaines suivantes des valeurs élevées de globulinémie persistent chez tous les animaux.

\section{Immunofluorescence (globulines totales)}

La moyenne géométrique des titres réciproques augmente à la $3^{\mathrm{e}}$ semaine de présence sur le terrain. Pour une même durée d'infection les anticorps anti-trypanosomiens sont plus élevés chez les Ndama que les zébus.

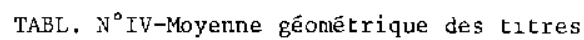
réciproques entre 40 et 48 jours.

\begin{tabular}{|c|c|c|c|c|c|c|}
\hline An imaux & $1 / 80$ & $1 / 160$ & $1 / 320$ & $1 / 640$ & $\mathrm{n}$ & MGTR \\
\hline N2 & 2 & 1 & 1 & 1 & 5 & 96,1 \\
\hline N1 & 5 & 2 & 2 & 6 & 15 & 141,6 \\
\hline ZN & 0 & 1 & 1 & 2 & 4 & 380,4 \\
\hline Z1 et Z2 & 5 & 5 & 1 & 1 & 12 & 77,81 \\
\hline
\end{tabular}

On notera que le groupe $\mathrm{ZN}$ a une production d'anticorps très élevée.

Il n'y a pas, au demeurant, de corrélation quantitative entre la présence d'anticorps et l'absence de mortalité. Tous les animaux qui ont succombé, parmi les Ndama, sont positifs avec des titres de positivité relativement élevés, entre $1 / 80$ et $1 / 640$ ). Qualitativement, il y a certainement des différences marquées entre les Ndama qui résistent et tous les autres animaux.

\section{Immunoglobulines}

La moyenne géométrique des titres réciproques d'immunoglobulines IgM n'augmente que légèrement chez les Ndama; chez les zébus, à partir de valeurs assez basses il y a, au moment de la parasitémie, une augmentation $(31,74)$ qui devient très importante après traitement, corrélativement à l'augmentation des globulines totales.

TABL. $N^{\circ}$ III-Globulines totales du sérun : moyennes en $g / 1$

\begin{tabular}{|c|c|c|c|c|c|}
\hline Journées & 6.6 .77 & 13.6 .77 & 27.6 .77 & 11.7 .77 \\
\hline N'Dama & $49,1 \pm 3,49$ & $50,7 \pm 5,59$ & $56 \pm \pm 3,75$ & $55,18 \pm 3,12$ & $64,68 \pm 7,13$ \\
\hline Zébus & - & - & $43,57 \pm 6,01$ & $47,50 \pm 4,54$ & $62,25 \pm 3,65$ \\
\hline
\end{tabular}


TABL, $\mathrm{N}^{\circ} \mathrm{V}$-Inmunoglobulines IgM.

\begin{tabular}{|c|c|c|c|c|c|c|c|c|c|}
\hline Animaux & P é r i od es & $1 / 1$ & $1 / 10$ & $1 / 20$ & $1 / 40$ & $1 / 80$ & $1 / 160$ & $\mathrm{n}$ & MGTR \\
\hline \multirow{5}{*}{ N'Dama } & N1 et N2 avant expērience & 1 & 0 & 6 & 5 & 6 & 2 & 20 & 38,22 \\
\hline & N1 N2 $2 N-13$ juin & 0 & 2 & 3 & 2 & 3 & 0 & 10 & 30,31 \\
\hline & N1. N2 $2 \mathbb{N}-27$ juin & 0 & 2 & 5 & 8 & 4 & 6 & 25 & 48,56 \\
\hline & N1 N2 ZN - 11 juillet & $a$ & 0 & 3 & 5 & 1 & 4 & 13 & 55,07 \\
\hline & N1 N2 $\mathrm{ZN}-31$ octobre & 0 & 1 & 1 & 0 & 11 & 5 & 20 & 51,61 \\
\hline \multirow{5}{*}{ Zêbus } & Z1 avant expérience & 2 & 3 & 2 & 2 & 0 & 0 & 9 & 9,73 \\
\hline & 22 avant expërience & 0 & 3 & 2 & 3 & $v$ & 0 & 9 & 14,33 \\
\hline & 21 et $z 2-11$ juillet & 0 & 2 & 2 & 2 & 3 & $o$ & 9 & $31,7+$ \\
\hline & 21 et $22-25$ juillet & 0 & 0 & 0 & 2 & 1 & 5 & 8 & 103,7 \\
\hline & 21 et $22-31$ octobre & 0 & 0 & 1 & 0 & 4 & I & 6 & 71,25 \\
\hline
\end{tabular}

Les réactions obtenues avec les $\mathrm{IgG}_{1}$ sont très peu nettes; celles avec les $\operatorname{IgG}_{2}$ le sont moins et on peut dire que cette fraction augmente au cours de la maladie aussi bien chez les taurins que les zébus.

\section{Comparaisons d'ordre clinique}

Il n'apparaît pas de différences notables dans les manifestations cliniques de trypanosomiase, lorsque celle-ci s'extériorise, selon qu'il s'agit des zébus ou des Ndama. Tout au plus peut-on faire une distinction entre la maladie aiguë et la maladie chronique chez les uns et les autres en tenant compte des délais entre l'apparition des premiers signes et de l'évolution fatale.

Il est intéressant de noter que 4 des génisses $\mathrm{Z} 2$ ont mis bas des prématurés; 2 sont mortes avant la mise bas et il y a un cas de mortinatalité ; des 4 veaux obtenus sur le terrain, l'un est mort au bout de 24 heures. Les 3 autres ont survécu sans contracter de trypanosomiase pendant 2 mois : 2 ont succombé malgré tout ; le dernier a contracté une trypanosomiase à $T$. vivax et en est mort. Les mères ont une production laitière presque nulle. Ces faits sont suffisamment éloquents pour confirmer que l'élevage de zébus est actuellement impossible dans les régions infestées de glossines.

\section{EFFET LETHAL DES TRYPANOSOMES : ETUDES D'ANATOMIE PATHOLOGIQUE ET HISTOPATHOLOGIQUE}

1) Il importe, avant d'analyser la léthalité dans les lots, de définir maladie aiguë et maladie chronique dans le cas particulier des trypanosomiases. L'infection aiguë doit être considérée comme celle qui résulte d'une parasitémie qui, quelle que soit la période de prépatence, a tendance à se maintenir, faible ou élevée, mais persistante, entraînant la mort dans des délais relativement courts. L'infection chronique, au contraire, est surtout caractérisée par une parasitémie, le plus souvent basse et intermittente, compatible avec de longs délais avant la mort. Entre ces 2 types, il convient de placer l'exacerbation de la maladie chronique ou le réveil de l'infection latente, événement pendant lequel la parasitémie devient persistante et léthale. Ces distinctions ont leur importance sur le plan anatomopathologique.

2) Quel que soit le type d'infection et le trypanosome en cause, nous constatons que la mort se produit en hypothermie.

3) Durant les heures qui précèdent la mort, le nombre de parasites baisse généralement et l'examen peut même être négatif.

4) Les mortalités suivantes ont été constatées comme directement liées aux trypanosomiases :
groupe $\mathrm{N} 1 \ldots \ldots \ldots .8$ animaux sur 18
groupe $\mathrm{N} 2 \ldots \ldots \ldots \ldots, 6$ animaux sur 6
groupe $Z N \ldots \ldots \ldots .2$ animaux sur 4
groupe $Z 1$ et $Z 2 \ldots \ldots \quad 13$ animaux sur 16

Il n'est pas tenu compte de 3 mortalités pour des raisons non évidentes, ni des veaux nés en cours d'expérimentation et morts par la suite.

5) Les lésions qui sont décrites ci-dessous sont, le plus souvent, la synthèse de plusieurs observations. 


\section{Phanères}

La maigreur accentue les escarres de décubitus car les os sont proéminents chez tous les animaux autopsiés.

\section{Carcasse et muscles}

La graisse de couverture est absente; il y a hydrose du tissu conjonctif sous cutané et hydrocachexie : muscles pâles et imbibés ; espaces aponévrotiques gélatineux.

\section{Rate}

1) Les lésions ne sont pas constantes et sont de plusieurs types :

a) splénomégalie : vraisemblablement de stase puisque la rate ne présente pas le caractère des rates de septicémie. Ici elle est grosse (jusqu'à $60 \mathrm{~cm}$ sur $19 \mathrm{~cm}$ chez les zébus et 51 sur $14 \mathrm{chez}$ les Ndama, l'épaisseur étant de 2 à $6 \mathrm{~cm}$ ), avec une capsule légèrement épaissie, une pulpe rouge consistante et des trabécules développés ; parfois présence de pétéchies sur la capsule, à la face interne. Cette altération est observée avec $T$. congolense et $T$. vivax chez les Ndama: maladie aiguë; avec $T$. vivax chez les zébus : maladie aiguë ;

b) rate de volume normal présentant des pétéchies capsulaires ( $T$. vivax chez les Ndama et les zébus ; maladie aiguë) ; pas d'écoulement à la coupe mais tuméfaction;

c) rate atrophique : plissement de la capsule, réduction de pulpes rouge et blanche, grande densité de trabécules ( $T$. vivax, évolution chronique chez les zébus).

2) A ces observations correspondent microscopiquement et dans l'ordre :

a) une pulpe rouge plus abondante que normalement; une atrophie folliculaire et un dépeuplement en lymphocytes ;

b) une image d'hyperhémie avec présence de nombreuses hématies entre les cellules lymphoïdes, mais sans masquer celles-ci ; la capsule et les trabécules ont des capillaires congestionnés ;

c) une hyperplasie réticulaire par collagénisation avec réduction de la pulpe rouge et des follicules.

Dans les 3 cas, il y a surcharge d'hémosidérine.

\section{Ganglions}

1) L'augmentation de volume des ganglions lymphatiques est chose courante chez les animaux de nos régions. Il y a cependant une hypertrophie ganglionnaire liée aux trypanosomiases et celle-ci est d'autant plus apparente que les animaux atteints sont très maigres. Les ganglions sont visibles d'assez loin. Avant expérimentation, aucun des animaux utilisés ne révélait de ganglions apparents ; à la palpation, les ganglions prescapulaires et précruraux glissent entre les doigts et leur mensuration est assez malaisée. Antérieurement à l'infection, les dimensions moyennes sont :

- prescapulaires : $8,6 \mathrm{~cm}$ pour les zébus, $9 \mathrm{~cm}$ pour les Ndama ;

— précruraux : $7 \mathrm{~cm}$ dans les 2 races.

Sur les animaux autopsiés, les ganglions prescapulaires mesurent entre 9 et $16 \mathrm{~cm}$, les précruraux entre 7 et $12 \mathrm{~cm}$. Si les mesures faites in vivo ont donné des valeurs proches de la réalité, alors il y a eu augmentation de la taille des ganglions au cours de la maladie. Ceux-ci sont toujours entourés de masse gélatineuse : hydrose du tissu conjonctif périphérique. La capsule porte quelquefois des pétéchies. On note une différence entre les infections aiguës et celles qui sont chroniques. Dans les premières, assez souvent, la congestion corticale est manifeste tandis qu'elle manque dans les secondes. Le plus souvent l'organe est succulent à la coupe. La chronicité semble davantage marquée par des ganglions plus fermes, moins succulents, grisâtres; dans quelques cas, on a noté des ganglions à coloration brune, traduisant des troubles dystrophiques hémosidériniques. Il ne semble pas y avoir de différences selon qu'il s'agit de zébus ou de Ndama, ni selon l'espèce de trypanosome ayant causé la mort. Il est remarquable qu'en plus des hypertrophies ganglionnaires, il $y$ a très souvent augmentation de la taille des ganglions hématiques médiastinaux et retropéritonéaux qui ont entre 0,5 et $1 \mathrm{~cm}$ de diamètre et semblent plus nombreux.

Les réactions ganglionnaires hypertrophiques et les disséminations ganglio-hémales les plus marquées sont observées le plus fréquemment chez les animaux morts de trypanosomiase à T. vivax aussi bien avec les zébus qu'avec les Ndama. 


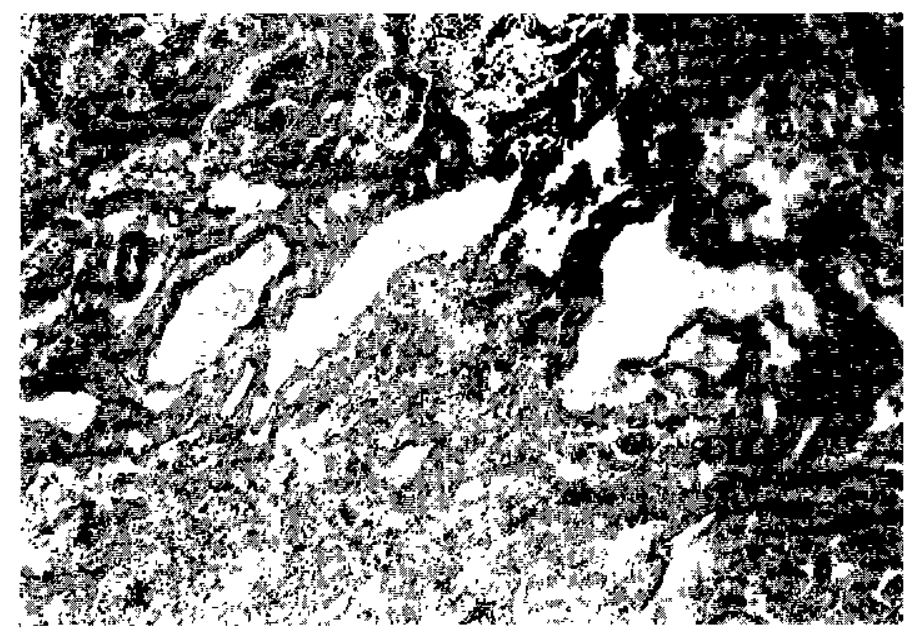

Photo. $\mathrm{n}^{0}$ 1. - Ganglion lymphatique de zébu; $T$. vivax, maladie chronique: prolifération collagène trabéculaire des sinus.

Photo, $\mathrm{n}^{\circ} 2$ - Fole de bovin Ndama : prépatence 32 jours ; séjour sur le terrain : 108 jours : triple infection $T$. vivax, $T$. congolense, $T$. brucei. Début de nécrose centrolobularre.

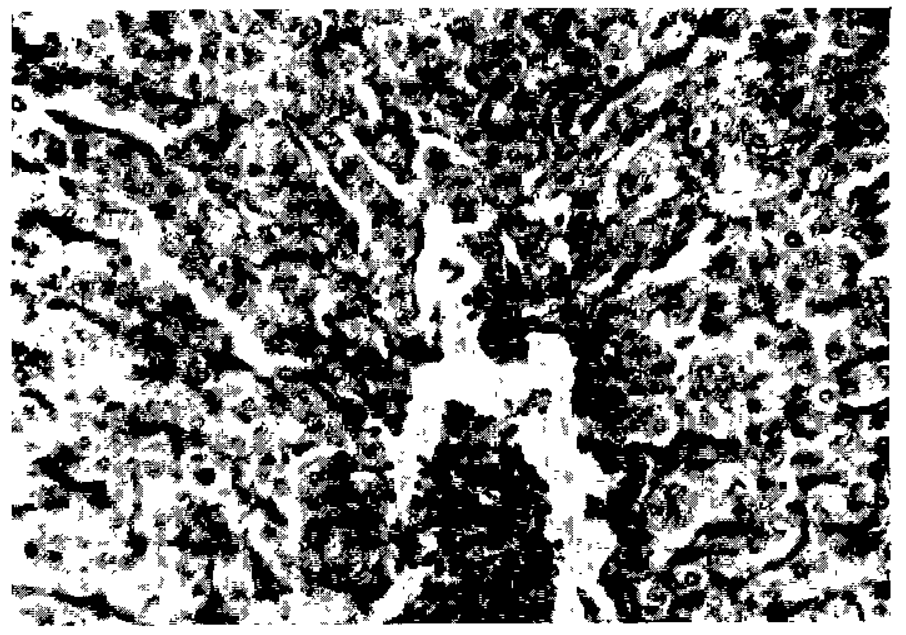

Photo. $\mathrm{n}^{\circ}$ 3. $\rightarrow$ Cour de Ndama : maladie aiguë due à $T$. vivax (19 jours); Myocardite avec destruction des fibres et invasion leucocytaire. 
Photo, $n^{\circ}$ 4. - Reın de bovin zébu atteint de maladie due à $T$. vivax (en 108 jours) : glomérulo-néphrite.
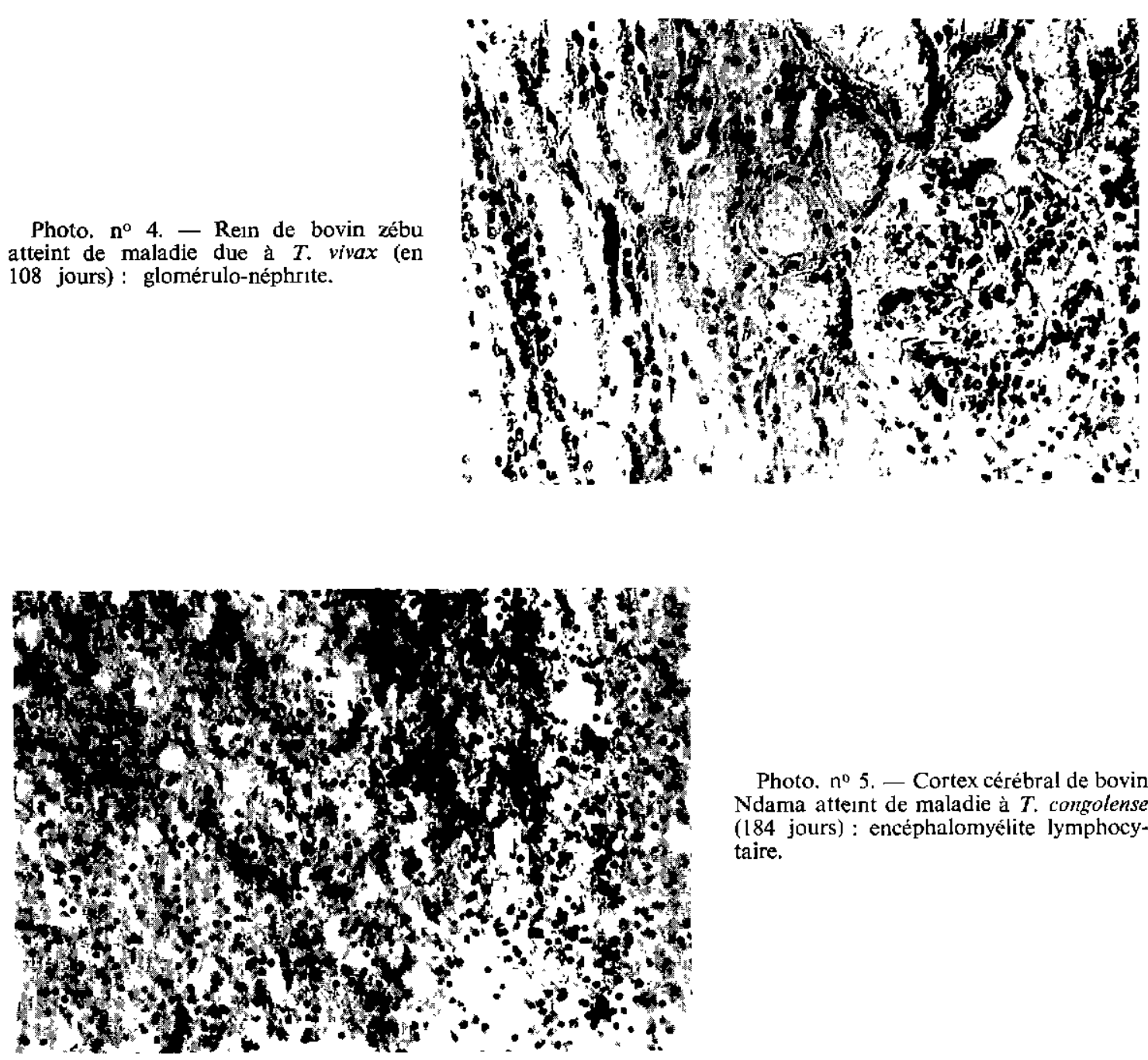

Photo. $\mathrm{n}^{0}$ 5. - Cortex cérébral de bovin Ndama atteint de maladie à $T$. congolense (184 jours) : encéphalomyélite lymphocytaire.

Photo. $n^{\circ}$ 6. - Calque de cœur de bovin Ndama atteint de maladie due à $T$. congolense (184 jours: agglutination et lyse des trypanosomes.

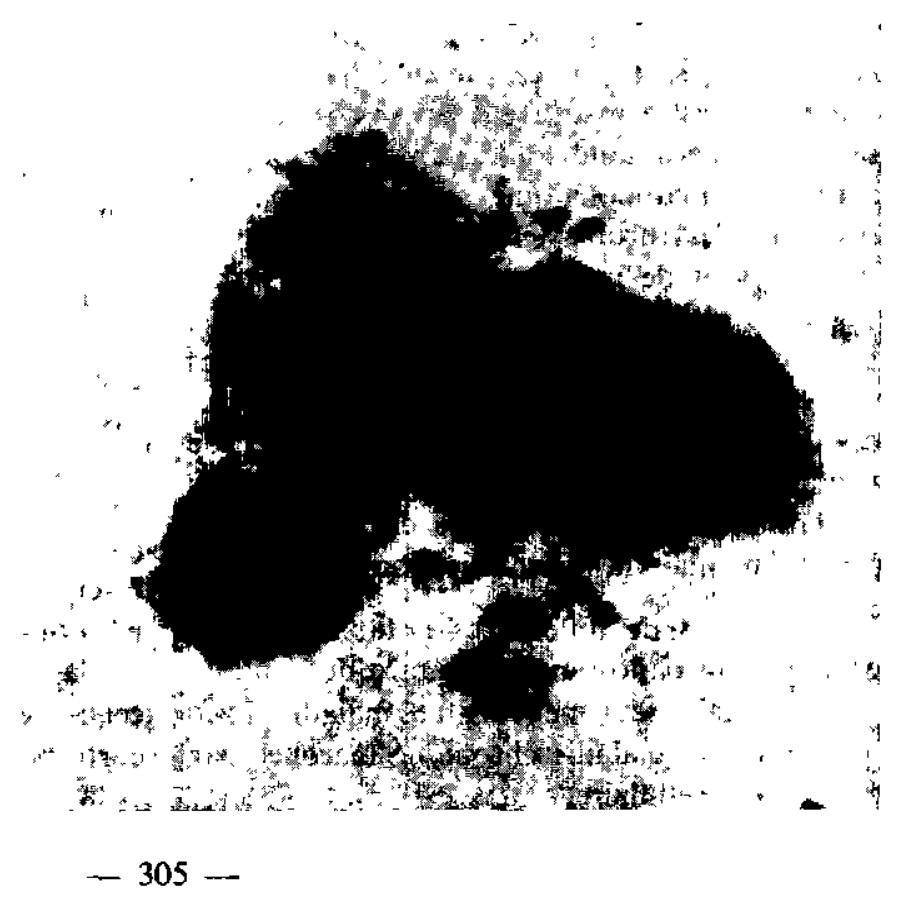


2) Microscopiquement, l'image prédominante est un changement dans la composition cellulaire du contenu ganglionnaire : il y a de nombreux lymphoblastes, cellules plasmatiques, macrophages et cellules polynucléées. Dans les infections aiguës, les nodules lymphatiques et les centres germinatifs sont peu affectés mais les sinus sont dépeuplés et les trabécules s'étendent. Quand la maladie évolue plus longtemps, les sinus sont envahis par des fibres collagènes. La collagénisation des trabécules est très accentuée dans les cas chroniques, accompagnée par une hypoplasie des nodules lymphatiques.

La présence de globules rouges dans la partie corticale et en dehors des sinus n'a été constatée que chez les animaux morts au bout d'un mois, au plus, après le début de la parasitémie.

\section{Moelle osseuse}

1) La moelle sternale et vertébrale reste rouge. Des modifications importantes surviennent au niveau des os longs. Une section fémorale révèle dans tous les cas une involution séro-gélatineuse de la moelle diaphysaire, souvent sur la plus grande partie, mais quelquefois limitée à la partie médiane quand il y a hématopoièse compensatoire. Les épiphyses restent rouges, sauf, parfois, dans les parties distales. Toutefois, on peut noter pendant les quelques premiers mois de maladie, une réactivation hématogène de la moelle.

2) L'étude histologique montre que la moelle diaphysaire, dans les cas avancés mais non chroniques, est constituée d'un endosteum de fibres collagènes, tandis que, dans les épiphyses, l'activité hématopoiétique se traduit par la présence d'érythroblastes, d'érythrocytes, de myélocytes et de mégacaryoblastes ; on y distingue, en plus grand nombre, pour ce qui est des cellules de la lignée blanche, des populations groupées de métamyélocytes granulocytes éosinophiles ou neutrophiles. Dans deux cas de maladie chronique, il n'y a que quelques rares lymphoblastes dans les trames de l'endosteum épiphysaire mais la vascularisation persiste.

Il n'y a pas de différences notables suivant l'espèce de trypanosome ou la race de l'animal parasité, mais seulement entre maladie avec régénération et maladie chronique marquée par l'épuisement cellulaire.

\section{Foie}

1) Les lésions du foie sont celles de congestion hépatique ( $T$. vivax/zébus, jusqu'à 2 mois) ou de dégénérescence ( $T$. vivax; $T$. congolense/zébus et Ndama) pour les évolutions plus longues. Dans quelques cas, il est observé une transition entre la congestion et la dégénérescence ( $T$. vivax/zébus). L'hypertrophie accompagne ces lésions : le foie est gros avec des bords arrondis.

La vésicule biliaire est souvent remplie de bile, quelquefois jusqu'à la distension.

2) Microscopiquement les altérations correspondent :

a) à une congestion discrète : les capillaires sinusoïdes sont remplis de sang mais les cellules hépatiques apparemment peu modifiées ;

b) à une nécrose centrolobulaire et atteinte des cellules hépatiques qui deviennent mal délimitées, avec des noyaux pycnotiques ;

c) à une modification fonctionnelle par mobilisation des cellules de Kupffer et invasion macrophagique, le tout s'accompagnant de dégénérescence de cellules hépatiques.

\section{Ceur}

1) Les lésions cardiaques sont constantes, qu'il s'agisse des zébus ou des Ndama, et quel que soit le trypanosome pathogène en cause.

Chez les animaux morts naturellement, l'arrêt du cour a lieu en diastole; les ventricules sont remplis de sang. Plusieurs types de lésions sont rencontrés :

a) myocardite congestive : volume du cour apparemment normal; lésions écchymotiques ou pétéchiales à localisation variable : épicardique ou endocardique, ventriculaire ou auriculaire ; l'auricule droite est atteinte dans tous les cas observés ; la veine cave antérieure est également atteinte et il y a aussi endocardite valvulaire. Ces lésions du cœur s'observent aussi bien chez les Ndama que les zébus infectés de $T$. vivax avec une parasitémie souvent élevée $(+++)$;

b) myocardite dégénérative : le volume du cœur est peu modifié; le myocarde est pâle, comme cuit; quelquefois des pétéchies très discrètes ; mais surtout l'épicardium a des trâ̂nées ou des marbrures jaunâtres bien au-dessus de la graisse sub-épicardiale; au demeurant, celle-ci est flasque et gélatineuse. A cette lésion correspond une maladie chronique chez les Ndama, due à $T$. congolense ou à $T$. vivax; 
c) hypertrophie cardiaque : le cœur mesure près de $22 \mathrm{~cm}$ sur 19 ; la pointe est arrondie ; le ventricule droit est dilaté; le sillon interventriculaire droit laisse apparaître la branche ventriculaire de la veine cardiaque qui est aussi dilatée de même que ses ramifications; à la coupe, la paroi ventriculaire droite est plus mince que normalement et le myocarde légèrement pâle ; pas de pétéchies évidentes. Le cœur hypertrophique a été observé plusieurs fois dans les formes aiguës de trypanosomiase à $T$. vivax chez les zébus et les Ndama.

2) Microscopiquement l'image prédominante est toujours la myocardite dans les 3 cas :

a) de type nécrotique, avec dissociation des fibres du myocarde, présence de foyers inflammatoires où apparaissent de nombreux leucocytes, infiltration hémorragique ;

b) de type dégénératif : fibres à noyaux pycnotiques et à striation peu marquée, plages de lyse cellulaire et invasion de macrophages et de lymphoblastes.

\section{Poumons}

1) Il est toujours anormal dans les cas considérés : pneumonie et emphysème; les lésions atteignent le plus souvent les 2 lobes et la pneumonie évolue jusqu'à l'hépatisation.

2) Microscopiquement : images de bronchopneumonie, d'emphysème associé à une bronchiolite.

\section{Reins et vessie}

1) Il est noté, assez souvent, une congestion corticale discrète dans la maladie aiguë due à T. vivax ; dans les autres cas, il s'agit de dégénérescence corticale et médullaire avec, parfois, des pétéchies capsulaires; la pyélonéphrite dégénérative se rapporte aux cas avancés de trypanosomiase chez les zébus comme les Ndama atteints de $T$. vivax ou $T$. congolense.

La vessie présente des pétéchies ou des ecchymoses chez les Ndama atteints de $T$. congolense. La réplétion urinaire voire la rétention est aussi fréquente.

2) Microscopiquement ces lésions se traduisent par :

a) une congestion par infiltration de globules rouges entre les tubuli contorti et autour des corpuscules glomérulaires ;

b) une dégénérescence des tubuli contorti et la présence de sérosité dans la lumière des tubules; celle-ci peut être poussée jusqu'à la desquamation des cellules tubulaires et leur lyse ;

c) une glomérulonéphrite proliférative de type subaigu.

\section{Cerveau}

1) L'atteinte cérébrale est irrégulière, du moins discrète macroscopiquement, dans la plupart des cas examinés. Il est constaté une congestion des capillaires méningés du cerveau et du cerebellum dans les formes aiguës de trypanosomiase à $T$. vivax chez les zébus. Chez 3 animaux, 2 Ndama et un zébu, atteints de $T$. vivax, une tuméfaction trouble est observée au niveau du cortex.

2) Microscopiquement la méningo-encéphalite est manifeste chez beaucoup des animaux, sans discrimination entre les espèces de trypanosomes ou les animaux atteints : les méninges et le cortex cérébral sont envahis par des lymphoblastes et des macrophages.

\section{Nerfs}

1) Le nerf sciatique, choisi comme témoin des atteintes névritiques, ne révèle rien macroscopiquement.

2) L'histologie, par contre, indique qu'il y a prolifération des fibres de l'épineurium et collagénisation, ce qui laisse de grandes distances entre les îlots de l'endoneurium ; mais apparemment les fibres de myéline et l'axon ne sont pas atteints ; il n'y a qu'une infiltration leucocytaire discrète dans le nerf.

\section{Réservoirs digestifs et intestins}

1) Les interprétations sont aléatoires du fait du polyparasitisme par Helminthes et Coccidies relaté plus haut. Néanmoins, dans les cas de trypanosomiase aiguê où le phénomène congestif est quasiment général, on est fondé à rattacher les lésions de congestion, observées au niveau des réservoirs, à la maladie étudiée : plages écchymotiques sur la muqueuse du rumen, de l'omasum et du réticulum. L'abomasum ainsi 
que le jejunum et le colon ont des lésions plus marquées avec de larges plages congestives. Le mésentère est souvent brunâtre avec, nous l'avons vu, une hypertrophie ou une prolifération des ganglions hématiques. Extérieurement, il y a congestion à la limite des anses intestinales et du mésentère. L'inflammation intéresse aussi le péritoine.

\section{Appareil reproducteur}

Les conclusions sont réservées. Certaines génisses ont mis bas normalement, sans trypanosomiase néonatale chez les veaux. Toutefois, les mamelles des mères sont restées affaissées, flasques, sans production notable de lait.

Chez le zébu mâle entier, la surface de l'albuginée testiculaire présente des ecchymoses. A l'bistologie, la spermatogenèse ne semble pas perturbée au niveau des cellules-souches, mais il y a peu de spermatozoïdes dans la lumière des tubes séminifères: maladie aiguë đue à T. vivax.

\section{Organes endocrines}

Les autopsies pratiquées sur le terrain n'ont pas isolé les thyroïdes, les surrénales, le pancréas, l'hypophyse et autres organes endocrines et il n'en est pas tenu compte ici. Il faudrait que d'autres études permettent de le faire, parallèlement à dosages d'hormones.

\section{Liquides cavitaires}

C'est à dessein que ce paragraphe est individualisé au lieu d'en traiter à propos de chaque organe car il intéresse la physiopathologie générale. Dans la maladie due à $T$. congolense comme celle due à $T$. vivax, aussi bien chez les zébus que chez les Ndama, l'atteinte chronique est accompagnée de présence de liquides dans les cavités: notamment hydropéricardite et ascite; les liquides sont séreux, légèrement ambrés; dans un seul cas ( $T$. congolense), il a été observé un liquide sérofibrineux dans le péricarde d'un Ndama atteint de maladie chronique.

\section{Présence de trypanosomes dans les organes et les liquides cavitaires}

Les autopsies pratiquées dans les premières heures qui suivent la mort naturelle ou sur les animaux sacrifiés permettent de constater la présence de trypanosomes dans les liquides et certains organes du corps.

D'abord le sang; le nombre de trypanosomes y est élevé ou au contraire bas. Des bêtes qui présentaient une forte parasitémie 2 jours avant la mort ont souvent un plus faible nombre de parasites lorsqu'elles agonisent.

Dans les cas d'hydropéricardite et d'ascite, il y a aussi des trypanosomes extravasculaires ; la cavité péritonéale renferme indifféremment $T$. vivax ou $T$. congolense. Les frottis par apposition à la surface des méninges révèlent des trypanosomes qu'on peut penser extravasculaires, les lames étant dépourvues d'hématies.

La démonstration de parasites dans les organes est malaisée sur des coupes histologiques. En effet, dans les foyers inflammatoires, l'existence de nombreux leucocytes masque la présence des parasites et, au demeurant, il est difficile de reconnaître ceux-ci. Pour ce qui est de $T$. congolense en particulier, la dissection de veinules de l'épicardium et du mésentère a souvent permis de constater la localisation intra-vasculaire de ce trypanosome; il en est de même concernant la microvascularisation du plexus choroïde. Sur les coupes histologiques de tous les organes, la tentation est souvent forte de dire qu'il y a des trypanosomes dans les tissus, à l'intérieur des capillaires, mais il est hasardeux de l'affirmer à partir des méthodes d'étude adoptées ici, à cause de la forme très allongée des noyaux des fibres de la musculature lisse qui borde certains capillaires et que la coupe peut entraîner dans les parenchymes. Les seules évidences, à la coloration par l'hématoxyline ou le glychémalun, sont liées aux plages nécrotiques dans le cœur où de nombreux granules métachromatiques se rapportent aux trypanosomes.

C'est d'ailleurs ce que confirme les calques d'organes colorés au May-Grünwald-Giemsa. Chez presque tous les animaux, il y a dans le cœur accumulation de trypanosomes, le plus souvent agglutinés et au voisinage de cellules de la lignée blanche; il y a aussi phagocytose très prononcée. Assez curieusement, chez beaucoup des bovins trypanosomés, on trouve dans le cœur Sarcocystis fusiformis en plus grand nombre que nous ne l'avons observé jusqu'à maintenant sur des animaux tout-venants. Les coupes sagittales de myocarde révèlent chez certaines bêtes des kystes assez gros, bourrés de ce parasite. La trypanosomiase favorise-t-elle 
la prolifération des sarcocystes ? Ces sarcocystes se retrouvent aussi, chez quelques animaux, dans la moelle rouge hématogène. Enfin, cette moelle est également, après le myocarde, le tissu qui renferme le plus de trypanosomes agglutinés ou phagocytés par des macrophages. Tous les autres organes, la rate, le foie, les ganglions, les reins, ne renferment, en comparaison, que très peu de trypanosomes dans leur parenchyme, voire, assez souvent, pas du tout, même chez les bovins fortement parasitémiques; les sinus sanguins ne sont pas pris en considération.

\section{Essai d'interprétation physiopathologique}

Il est logique de penser que les trypanosomiases animales, qu'elles soient dues à $T$. congolense ou à $T$. vivax, sont avant tout une maladie qui résulte essentiellement de l'atteinte du tissu sanguin et, secondairement, de dommages provenant des réactions de l'organisme visant à pallier les altérations du sang et à en juguler la cause.

En effet, la perturbation initiale est l'anémie par destruction des globules rouges. Elle est de type inflammatoire. L'absence d'hémoglobinurie fait retenir qu'à la phase initiale, ce qui prédomine, c'est l'hémorragie interstitielle (congestion passive observée dans les organes, suivie d'hémolyse et de surcharge hémosidérinique), et c'est aussi la phagocytose globulaire extravasculaire par les macrophages du système réticulohistiocytaire. L'anémie est au début normochrome et normocytaire. Elle est régénérative comme en témoigne l'hyperplasie érythropoiétique compensatrice de la moelle osseuse. Les désordres s'amendent plus ou moins dans les cas chroniques et en particulier chez le Ndama où l'anémie, très souvent, n'est sévère qu'au bout de plusieurs mois, si l'animal est appelé à mourir de sa maladie. Dans les autres cas qui concernent tous les zébus et une partie des Ndama, l'anémie régénérative est suivie d'une phase consécutive à l'insuffisance médullaire due à une hyperdestruction qui dépasse les possibilités de compensation. L'anémie observée est alors microcytique et hypochrome. Il n'est pas rare d'observer, à la limite, une anisocytose.

Parallèlement à la destruction érythrocytaire, les trypanosomes, à l'endroit où ils se trouvent, entraînent des lésions. La localisation est mal définie en ce qui concerne $T$. vivax, mais elle est intravasculaire s'il s'agit de $T$. congolense. Quoi qu'il en soit, les trypanosomes provoquent une inflammation qui, macroscopiquement, n'entraîne pas de nécrose marquée ; car il n'y a ni perte de substance dans les parenchymes, ni formation d'abcès, ni calcification. Sans doute peut-on retenir dans plusieurs cas une vascularite préalable, avec infarcissement et mobilisation de cellules de la lignée blanche pour la destruction des trypanosomes. Il en résulte, à la longue, des troubles circulatoires. Dans les cas avancés, le cœur est hypertrophié et présente une insuffisance cardiaque droite. C'est certainement ce qui explique l'œdème du cerveau ou bien la congestion passive des veines, l'hypertrophie de la rate par stase, ainsi d'ailleurs que celle du cœur. Plusieurs organes se trouvent ainsi atteints. L'augmentation du volume du cœur procède d'une dilatation fonctionnelle et non d'une hypertrophie cellulaire.

La lutte contre la septicémie trypanosomienne et la destruction parallèle des globules rouges demande de gros efforts à tous les tissus du système réticulo-histiocytaire : il en résultera une aplasie de la moelle et une insuffisance médullaire globale se traduisant, en fin d'évolution, par une pancytopénie et un coma anémique, puis une défaillance cardiaque. Cette dernière peut aussi bien être liée à une réaction de type anaphylactique provoquée par des réactions antigènes-anticorps conduisant, dans cette maladie, à une vasoconstriction des artères pulmonaires et la mort par défaillance du cœur droit. Survenue de la sorte, la léthalité n'est pas incompatible avec une valeur hématocrite élevée, quand les possibilités de régénération n'ont pas été épuisées.

Les phénomènes de stase aboutissant à l'atrophie ne sont que rarement observés car les animaux meurent avant. Cependant, la rate de zébu chroniquement atteint est volontiers hypoplasique.

Pour résumer, c'est l'atteinte du sang et les répercussions sur les vaisseaux et sur les organes du S. R. E. ainsi que les implications immunitaires qui, en fin de compte, font que les trypanosomiases sont une maladie léthale.

On ne saurait cependant s'en tenir à un ou deux schémas physiopathologiques car il y a certainement plusieurs modalités dans le détail, suivant que la maladie est aiguë ou chronique, qu'elle est due à telle ou telle espèce ou souche de trypanosome, qu'elle atteint telle ou telle race animale, que les malades sont un terrain neuf ou 
antérieurement infecté, qu'il y a ou non un stress surajouté pouvant modifier le cours de la maladie. En vérité, malgré tout ce qui a été dit et écrit, beaucoup de travaux restent à faire pour élucider le mécanisme des trypansomiases. Bien entendu, il faut ajouter que les hypothèses, présentement, ne sont que des moyens pour atteindre des certitudes même si elles contiennent des contradictions.

\section{COMPARAISONS ENTRE ZEEBUS ET NDAMA}

\section{Maladie due à Trypanosoma vivax}

\section{1) Forme aiguë}

\section{a) Zébus}

Le zébu sahélien, jamais en contact avec des glossines, développe une maladie aiguë dès les premières semaines de l'infection par des trypanosomes. La période de prépatence est courte : une à 2 semaines; la parasitémie est élevée et souvent persistante; les signes cliniques sont d'emblée sévères : anémie, amaigrissement, hyperthermie, réactions ganglionnaires hyperplasiques, larmoiement, jetage, dyspnée, inappétence, incoordination motrice, décubitus, en fin de compte, et mort par coma anémique et défaillance cardiaque. On n'y peut rien, sauf à traiter les animaux par trypanocide.

\section{b) Ndama}

Il nous faut admettre que les Ndama n'ayant jamais vécu dans une région infestée de glossines développent une maladie aiguë mais à évolution un peu plus longue que chez les zébus. L'issue est la même pour plusieurs animaux : elle est fatale. Une conséquence très importante en découle : il ne faut pas élever du bétail Ndama dans des régions indemnes de trypanosomiase pour les exporter dans des contrées endémiques. Sinon il y aura dans les premiers temps un pourcentage important de morts par maladie aiguë, avec des signes cliniques et des détériorations organiques semblables à ce qui est observé chez les zébus.

\section{2) Forme chronique}

a) Zébus

La chronicité est induite par une maladie antérieure qui a été traitée. Dès que cessent les effets du médicament utilisé, les trypanosomes se développent, en grand nombre ou en faible nombre, mais la maladie suit son cours et les délais qui conduisent à la mort peuvent être de plusieurs semaines ou quelques mois mais l'issue est presque toujours fatale.

\section{b) Ndama}

Une partie des survivants, ceux qui ont passé le cap de la maladie aiguë, restent trypanosomés. C'est ici que l'on perçoit des différences minimes d'avec les zébus. La parasitémie est inconstante, la température irrégulièrement élevée, l'anémie régénérée puis, à d'autres moments, à nouveau provoquée. Les signes cardinaux de trypanosomiase sont inconstants mais les bêtes sont maigres, adynamiques et dans un état de santé très précaire. Quand les principaux organes du S. R. E., trop souvent sollicités pour lutter contre les anémies successives et les vagues septicémiques, n'en peuvent plus, il s'ensuit une pancytopénie et une défaillance cardiaque. Etat de santé aléatoire aussi, du fait qu'un stress quelconque peut déclencher une flambée aiguë sur un fond de chronicité.

Cette chronicité, n'est-ce pas ce qui est corrélé à la trypanotolérance ? En effet, la trypanotolérance n'est autre que l'aptitude des Ndama à survivre tout en étant infectés: prémunition cependant aléatoire chez beaucoup de bêtes. Il y a lieu de réfuter catégoriquement la notion d'innocuité car il y a réellement des dommages organiques qui ne sont pas sans abaisser les performances zootechniques.

\section{Maladie due à Trypanosoma congolense}

\section{a) Zébus}

Le nombre très limité de cas dans la présente expérience ne permet pas de donner des conclusions aussi catégoriques que ci-dessus, mais d'autres auteurs ont étudié ce cas particulier sur un plus grand nombre d'animaux.

\section{b) Ndama}

La présente expérimentation n'a pas permis, encore une fois, de bien étudier cette maladie mais des études antérieures montrent une plus grande fréquence de cas chroniques de trypanosomiase : faible parasitemie, signes cliniques assez discrets, mais $T$. congolense est très anémiant chez les Ndama : anémie toutefois inconstante et régénérative. Ce qui a été dit plus haut 
sur les risques que comportent la maladie chronique due à $T$. vivax vaut aussi pour $T$. congolense. Cependant, l'écologie et la physiopathologie du bétail Ndama atteint de $T$. congolense demande, à notre avis, des études fondamentales poussées, car selon nous, dans ce cas particulier, à la maladie chronique peut faire suite un état quasi-réfractaire aussi bien à l'égard de $T$. congolense lui-même que $T$. vivax.

\section{Absence de maladie}

Il est très important de souligner que 9 bovins, sur 18 des Ndama achetés dans la région de Diourbel et dont nous avions quelque doute quant à la provenance, n'ont, à aucun moment, révélé de trypanosome : ni sur frottis et gouttes épaisses, ni par la technique de WOO, ni même par la méthode de LANHAM par examen du culot de centrifugation de l'éluat obtenu à partir de sang filtré sur colonne de D. E. A. E.-cellulose. Ces animaux ont quelque peu maigri durant les 2 premières semaines passées sur le terrain puis pendant toute la durée de l'expérience ont eu une excellente conformation sans présenter aucun signe de maladie. L'anémie a été discrète à la première quinzaine puis s'est rétablie. A titre indicatif, les valeurs suivantes se rapportent à des hématocrites moyennes successives : $37,28,36,33,37,39,40,41,40,44$, 43 etc. Deux de ces animaux, autopsiés, ne montrent aucune lésion organique majeure qui signe une maladie. Que dire alors en conclusion sinon que certains Ndama, pas tous, à la suite de circonstances à élucider et de mécanismes à connaître parfaitement, résistent très solidement à la trypanosomiase. Cela indique la nécessité d'études de zootechnie et de physiopathologie pour sélectionner la résistance et pour savoir comment l'induire chez les êtres vivants qui sont normalement très réceptifs.

\section{DISCUSSION}

La connaissance de ce qui est normal, pour le comparer à ce qui ne l'est pas, est imparfaite en ce qui concerne les animaux domestiques de nos régions car il n'y a pratiquement pas d'animaux parfaitement sains et bien nourris. Les comparaisons que nous pouvons faire sont donc relatives et se rapportent à des animaux supposés normaux, opposés à d'autres, manifestement malades. Les analyses faites par D. FRIOT et H. CALVET (5) sont un fondement solide pour ces comparaisons en raison de leur précision et de leur grand nombre. Ces auteurs trouvent, sur 2199 données, que la moyenne générale de l'hématocrite est égale à $39,1 \mp 0,3$; les moyennes partielles varient plus ou moins selon les régions et les saisons, selon les races bovines et les classes d'âge. Pour nous en tenir aux comparaisons présentes, la moyenne chez les zébus et les métis Diakoré $(37,5 \mp 0,9 ; 37,7 \mp 0,2)$ est significativement plus élevée que pour les Ndama $(34,7 \mp 1,2)$. Les valeurs que nous avons obtenues avant expérimentation sont nettement supérieures à celles-ci et nous ne les tenons pas pour la normale. Récemment, sur un effectif de 355 Ndama de région infestée de glossines, nous avons trouvé une moyenne de 31,50 seulement.

Revenons sur quelques-unes des altérations relevées au cours de la présente expérimentation. Qu'il s'agisse des zébus ou des Ndama, une valeur de l'hématocrite inférieure à 20 peut conduire à une mort rapide. Des valeurs très basses (10 à 17) se rapportent souvent à un coma anémique. F. VOHRADSKY constate une hématocrite égale ou inférieure à 15 chez des animaux morts moins de 9 semaines après l'infection par $T$. vivax (15). Dans le cas de $T$. congolense, G. J. LOSOS et al. (9) trouvent $11,0 \mp 3,3$ au moment de la mort. Cependant, dans certains cas, nous avons constaté la mortalité avec des valeurs hématocrites plus élevées.

- 1 Ndama : $32($ T. vivax ++$)$

- 1 Ndama : $30(T$, vivax +$)$

- 1 Ndama : $30(T$. vivax,$+ T$. congolense ,+ T. brucei + )

- 1 Ndama : 27 (T. vivax +++$)$

- 1 Ndama : 27 ( $T$. congolense + ).

Tout bien pesé, la mort peut donc survenir quand l'hématocrite est encore élevée chez les Ndama : L'anémie, cependant, occupe une place importante dans la maladie et de nombreuses autres expériences l'attestent, comme celles de M. MURRAY et al. (10) qui sont des plus récentes, menées en Gambie, dans une zone écologique assez similaire à celle qui est la nôtre ici :

En récapitulant les résultats, il n'est constaté que peu de différences pathologiques chez les animaux malades ou qui meurent de trypanosomiase et $\mathrm{ce}$, indépendamment de l'espèce. $\mathrm{Au}$ demeurant, certains aspects pathologiques ont 
fait l'objet de synthèses assez précises : G. CURRASSON (3) ; G. J. LOSOS et B. O. IKEDE (8) et il n'est pas indispensable de s'étendre là-dessus dans cette note. Mais insistons qu'il y a des lésions nerveuses dans les trypanosomiases animales $(8,6)$.

L'augmentation des globulines totales et plus particulièrement des IgM est signalée par de nombreux auteurs, sans grandes différences quant aux races animales ou quant aux trypanosomes qui causent l'infection.

La pathogénie repose encore sur des hypothèthèses, mais plusieurs de celles-ci sont en passe d'être vérifiées, notamment pour ce qui est des atteintes vasculaires. On lira avec profit la mise au point de P. F. L. BOREHAM (2) qui montre le rôle joué par les kinines plasmatiques, facteurs médiateurs de l'inflammation et du choc anaphylactique et dont l'augmentation est remarquable lors des accès parasitémiques. Ces substances participent à la genèse des lésions vasculaires. Pour ce qui est de l'anémie, R. N. T. FIENNES (4) distingue 2 stades dans les infections dues à $T$. congolense et $T$. vivax: la phase aiguë avec des accès critiques et la phase de chronicité post-critique. Dans la première, il est observé une anémie macrocytaire, tandis que la chronicité est marquée par une anémie microcytaire. Lorsque la maladie se déroule sur un mode suraigu, l'hémolyse est sévère et s'accompagne d'hydrémie; elle est alors rapidement mortelle. La nature hémolytique de l'anémie est confirmée par de nombreux auteurs dont, récemment, D. I. SAROR et E. H. COLES (11) qui, travaillant sur 12 zébus, trouvent que chez ces bêtes l'hémolyse est extravasculaire et se produit dans les organes du système réticulo-endothélial; ces zébus étaient infectés de $T$. vivax par la seringue.

Quand on s'interroge sur les différences entre les groupes expérimentaux, la conclusion immédiate est qu'il n'y a pas de grande différence de sensibilité entre des animaux qui n'ont jamais été parasités par les trypanosomes : zébus et Ndama meurent, à plus ou moins long terme, avec les mêmes lésions organiques. On peut ajouter que les zébus même traités restent sensibles et sont voués à la mort dès que cessent les effets thérapeutiques. Certains Ndama, dont malheureusement nous ne connaissons pas le passé avec certitude, résistent parfaitement. Pourquoi et comment? De nombreuses hypothèses (13) sont à vérifier et d'autres à imaginer.

\section{REMERCIEMENTS}

Le service de Parasitologie du L. N. E. R. V. adresse ses remerciements :

- à la Direction de l'I. L. R. A. D. qui a obligeamment financé ces études ;

- à la Direction de la Santé et des Productions animales du Sénégal pour avoir mis à la disposition du projet un ingénieur des travaux d'élevage qui s'est acquitté de ses tâches avec beaucoup de compétence et de dévouement;

- au Service de Physiologie, Biochimie et Nutrition du L. N. E. R. V. qui a procédé à des analyses biochimiques;

- au chef du village de Missira qui nous a bien aimablement reçus et a facilité notre travail.

\section{SUMMARY}

Experiment on comparative pathology of zebu and N'Dama cattle naturally infected by pathogenic trypanosomes

The authors studied $T$. vivax and $T$. congolense trypanosomiases on some zebu and N'Dama cattle which had never been previously infested and which were exposed to natural infestation in a zone where the distribution of Glossina morsitans submorsitans and $G$. palpalis gambiensis was very dense.

Comparing the evolution of the affections and the lesions observed in both species, their main conclusion is that, if zebu cattle are very sensitive to trypanosome infection, N'Dama cattle which have never been previously in contact with infested glossina are also susceptible to some extent. Some N'Dama cattle display a remarkable immunity and these animals should be the subject of thorough immunological as well as genetic studies. Such animals could be selected or crossbred in order to create breeds naturally and totally trypanotolerant. 


\section{RESUMEN}

\section{Experiencia de patologia comparada entre bovinos cebu y Ndama sometidos} a la infección natural por tripanosomos patogenos

Los autores estudiaron la evolución de tripanosomiasis con $T$. vivax y $T$. congolense sobre cebues y Ndama no teniendo nunca hospedado dichos parásitos después de una exposición à la infestación natural en una región dónde se encuentran numerosas Glossina morsitans submorsitans y $G$. palpalis gambiensis.

Comparan la evolución de las afecciones así provocadas y las lesiones observadas. De ello concluyen que sı los cebues son muy sensibles para con la tripanosomiasis, los Ndama igualmente hasta cierto punto, aunque previamente no se pongan nunca en contacto con glosinas infestadas. Ciertos $\mathrm{Nda}$ mas tienen una resistencia excelente.

Dichos animales merecen un estudio inmunologico detenido asi como estudios geneticos por que se puede utilizarles, seleccionados y cruzados, para crear origines naturalmente y totalmente tripanoresistentes.

\section{BIBLIOGRAPHIE}

1. AMBROISE-THOMAS (P.). Etude séro-immunologique sur dix parasitoses par les techniques d'immunofluorescence. Lyon, Institut de Médecine et d'Hygiène tropicales, $1969.645 \mathrm{p}$.

2. BOREHAM (P. F. L.). Pathogénie des trypanosomes. Colloque sur les moyens de lutte contre les trypanosomes et leurs vecteurs, Paris, 12-15 mars 1974, p. 283.

3. CURASSON (G.). Traité de protozoologie vétérinaire et comparée. I. Trypanosomes. Parıs, Vigot Frères, 1943, 445 p, pl. h. t.

4. FIENNES (R. N. T. W.). Haematological studies in trypanosomiasis of cattle Vet. Rec., 1954, 66: 423434.

5. FRIOT (D.), CALVET (H.). Biochimie et elevage au Sénégal. Rev. Elev. Méd. vét. Pays trop., 1973, 26 (4) : 75a-98a.

6. KALINER (G.). Trypanosoma congolense, II. Histopathologic findings in experimentally infected cattle. Expl. Parasit., 1974, 36 (1) : 20-26.

7. LISON (L.). Statistique appliquée à la biologie expérimentale, Paris, Gauthier-Villars, 1968, 346 p.

8. LOSOS (G. J.), IKEDE (B. O.). Review of pathology of diseases in domestic and laboratory anımals caused by Trypanosoma congolense, $T$. vivax, $T$. brucei, T. rhodesiense and T. gambiense. Vet. Path., 1972, 9 (suppl.) $71 \mathrm{p}$.

9. LOSOS (G. J.), PARIS (J.), WILSON (A. J.), DAR (F. K.). Pathologie de la maladie du bétail causee par
Trypanosoma congolense. Bull. Epizoot. Afr., 1973 21 (3) : 239-249.

10. MURRAY (M.) et al. Cattle diseases in the Gambia. I. Clinical studies. II. Pathology studies (communication personnelle de travaux sous presse, 1977).

11. SAROR (D. I.), COLES (E. H.). Observations on Trypanosoma vivax induced anaemia in cattle. 15th meeting of Int. Scient. Council Trypanosomiase Research and Control, Banjul, 1977 (sous presse).

12. SCHALM (O. W.). Veterinary hematology. London, Baillère, Tindall \& Cassell, 1td, 1965. 664 p., réédité 1975.

13. TOURE (S. M.). La trypanotolérance : revue de connaissances. Rev. Elev. Méd. vét. Pays trop., 1977, 30 (2) : 157-174

14. TOURE (S. M.). Valeur de la méthode d'immunofluorescence indirecte dans le diagnostic des trypanosomiases bovines et leur étude épizootiologique. Rev. Elev. Méd. vét. Pays trop., 1975, 28 (4) : 463-472.

15. VOHRADSKY (F.). Clinical signs, daily rate of infection, physical changes in cattle artificially infected by Trypanosoma vivax. Rev. Elev. Méd. vét. Pays trop., 1971, 24 (2) : 251-263.

16. WOO (P. T. K.). A technique for the parasitological diagnosis of african trypanosomiasis. Trans. r. Soc. trop. Med., 1971, 65 (2) : 249.

17. WOO (P. T. K.). A statistical study of the sensitivity of the haematocrit centrifuge technique in the detection of trypanosomes in blood. Trans. r. Soc. trop. Med., 1974, 68 (4) : 319-326. 